\title{
Heritability of Magnetoencephalography Phenotypes Among Patients With Genetic Generalized Epilepsy and Their Siblings
}

Christina Stier, MSc, Adham Elshahabi, MD, MSc, Yiwen Li Hegner, MD, PhD, Raviteja Kotikalapudi, PhD, Justus Marquetand, MD, Christoph Braun, PhD, Holger Lerche, MD, and Niels K. Focke, MD

Neurology ${ }^{\circledR}$ 2021;97:e166-e177. doi:10.1212/WNL.0000000000012144

\author{
Correspondence \\ Dr. Focke \\ niels.focke@ \\ med.uni-goettingen.de
}

\begin{abstract}
Objective

To assess whether neuronal signals in patients with genetic generalized epilepsy (GGE) are heritable, we examined magnetoencephalography resting-state recordings in patients and their healthy siblings.

\section{Methods}

In a prospective, cross-sectional design, we investigated source-reconstructed power and functional connectivity in patients, siblings, and controls. We analyzed 5 minutes of cleaned and awake data without epileptiform discharges in 6 frequency bands $(1-40 \mathrm{~Hz})$. We further calculated intraclass correlations to estimate heritability for the imaging patterns within families.
\end{abstract}

\section{Results}

Compared with controls $(n=45)$, patients with GGE $(n=25)$ showed widespread increased functional connectivity ( $\theta$ to $\gamma$ frequency bands) and power ( $\delta$ to $\gamma$ frequency bands) across the spectrum. Siblings $(n=18)$ fell between the levels of patients and controls. Heritability of the imaging metrics was observed in regions where patients strongly differed from controls, mainly in $\beta$ frequencies, but also for $\delta$ and $\theta$ power. Network connectivity in GGE was heritable in frontal, central, and inferior parietal brain areas and power in central, temporo-parietal, and subcortical structures. Presence of generalized spike-wave activity during recordings and medication were associated with the network patterns, whereas other clinical factors such as age at onset, disease duration, or seizure control were not.

\section{Conclusion}

Metrics of brain oscillations are well suited to characterize GGE and likely relate to genetic factors rather than the active disease or treatment. High power and connectivity levels cosegregated in patients with GGE and healthy siblings, predominantly in the $\beta$ band, representing an endophenotype of GGE. 


\section{Glossary}

FWE = familywise error correction; GGE = genetic generalized epilepsy; GSWD = generalized spike-wave discharges; ICC = intraclass correlation; JME = juvenile myoclonic epilepsy; $\mathbf{M E G}=$ magnetoencephalography; SNR = signal-to-noise ratio.

Idiopathic/genetic generalized epilepsy (GGE) is a common epilepsy syndrome accounting for $15 \%-20 \%$ of all epilepsies. ${ }^{1}$ Different seizure types can occur, including absence, myoclonic, and generalized tonic-clonic seizures. ${ }^{2}$ For GGE, a polygenic background is presumed. ${ }^{3}$ So far, gene discovery has been scarce despite high heritability ${ }^{4}$ and large-scale collaborative efforts. ${ }^{3,5}$ Thus, it is of high interest to seek subclinical traits of the syndrome (endophenotypes) that reflect the genetic background of the disease and cosegregate in families with affected individuals. ${ }^{6}$ Candidate markers for GGE have been proposed, such as cognitive functioning, ${ }^{7-9}$ frontal lobe ${ }^{10}$ and hippocampal morphology, hippocampal function, ${ }^{11}$ and functional network topology. ${ }^{12}$ Furthermore, patients with juvenile myoclonic epilepsy (JME) and their siblings have shown increased activation of the motor system during cognitive tasks. ${ }^{13,14}$ However, increased brain connectivity and power has also been found in absence of cognitive load in a mixed GGE cohort and in widespread regions. ${ }^{15,16}$ It is less clear to which extent observed findings reflect disease activity, effects of seizure burden, or treatment. Also, various methodologic approaches hinder reproducibility and comparability of functional network studies. ${ }^{17}$ Given that characteristics of spontaneous brain oscillations at rest are heritable, ${ }^{18,19}$ the studies of unaffected siblings may help to disentangle genetic factors from secondary disease effects.

This study set out to assess whether imaging metrics based on oscillatory neural activity and measured by magnetoencephalography (MEG) during resting-state could represent an endophenotype of GGE.

\section{Methods}

\section{Standard Protocol Approvals, Registrations, and Patient Consents}

This study was approved by the local ethics committee of the Medical Faculty of the University of Tübingen and conducted in compliance with the principles of the Declaration of Helsinki. All participants provided written informed consent.

\section{Recruitment}

Patients with GGE and their siblings were consecutively recruited through the clinical database of the Department of Neurology, University Hospital of Tübingen, Germany, between 2013 and 2019. Advertisement and recruitment of controls was conducted in the local area. All patients were diagnosed with GGE according to the recent International League Against Epilepsy classification. ${ }^{2}$ At the time of the study, siblings and controls never had experienced seizures, were free of any neurologic and psychiatric diseases, and did not take any medication.

\section{MEG Recording}

Resting-state data were measured in supine position (275 channels system, CTF Inc.) in the MEG center of the University of Tübingen ( $585.9 \mathrm{~Hz}$ sampling rate). Participants underwent 30 minutes of continuous recording in order to have sufficient data after exclusion of segments with generalized spike-wave discharges (GSWD). All participants were instructed to relax, to keep their eyes closed, not to fall asleep, and not to think of anything in particular.

\section{Individual Head Anatomy}

A sagittal high-resolution T1-weighted image was acquired for all participants (3D-MPRAGE, repetition time $=2.3$ seconds, echo time $=3.03 \mathrm{~ms}$, flip angle $=8^{\circ}$, voxel size $=1 \times 1 \times$ $1 \mathrm{~mm})$, either on a Siemens Magnetom Trio 3T scanner equipped with a 12 -channel head coil (11/45 controls, $5 / 25$ patients) or at the Siemens Magnetom Prisma 3T system (Siemens AG) with a 64-channel head coil (34/45 controls, 18/18 siblings, 20/25 patients). Detailed description of processing methods and references can be found elsewhere. ${ }^{20}$ In brief, individual cortical surfaces were reconstructed using FreeSurfer (surfer.nmr.mgh.harvard.edu/) and further subjected to SUMA (afni.nimh.nih.gov/download/). SUMA decimated each participant's cortical surface to 1,002 common vertices per hemisphere. The surface was resampled using the fsaverage template (FreeSurfer) and SUMA (ld = 10). In addition, 6 subcortical nuclei (bilateral amygdala, hippocampus, thalamus, caudate, putamen, and pallidum) were reconstructed based on the fsaverage template. Each region was converted to surfaces and spatially normalized to Montreal Neurological Institute space (DARTEL; SPM12; fil. ion.ucl.ac.uk/spm/software/spm12/) using CAT12 DARTEL template (neuro.uni-jena.de/cat/). This procedure eventually yielded 2,338 vertices for each participant and point-for-point anatomical correspondence for cortical and subcortical regions. Finally, the individual cortical mesh was realigned to the CTF sensor space using the fiducial positions recorded during the MEG session. A volume conduction head model was constructed for the MEG source analysis using the single shell method implemented in Fieldtrip.

\section{MEG Data Processing and Source Analysis}

Preprocessing and further analysis steps were performed using Fieldtrip (fieldtriptoolbox.org/) running in MatLab (version 9.0, R2016a, Mathworks Inc.) as described and referenced elsewhere. ${ }^{20}$ In short, data were preprocessed (Butterworth band-pass filter $1-70 \mathrm{~Hz}$, line-noise removal), downsampled $(150 \mathrm{~Hz})$, and cut into epochs of 10 seconds 
length. Trials with GSWD were manually marked and excluded from the further analysis including one trial preceding and one trial after the event ( \pm 10 seconds). Each trial was visually inspected and trials with artefacts were manually removed (e.g., movements, excessive muscle activity, sensor jumps). We used independent component analysis to detect and manually reject cardiac and eye movement artefacts. All trials were again reviewed and vigilance was rated according to sleep scoring criteria of the American Academy of Sleep Medicine. Thirty trials of cleaned and awake data (300 seconds) per participant were randomly selected for source analysis. We performed spectral analysis on the MEG sensor data using a multitaper fast Fourier time-frequency transformation approach with frequency-dependent discrete prolate spheroidal sequences tapers for 6 frequency bands $(\delta: 2 \pm$ $2 \mathrm{~Hz}, \theta: 6 \pm 2 \mathrm{~Hz}, \alpha: 10 \pm 2 \mathrm{~Hz}, \beta 1: 16 \pm 4 \mathrm{~Hz}, \beta 2: 25 \pm 4 \mathrm{~Hz}$, and $\gamma: 40 \pm 8 \mathrm{~Hz}$ ). Power and the cross-spectral density were derived from the Fourier transformed sensor-level data. We used beamforming (dynamic imaging of coherent sources) to project the data to the source space. For each vertex point of the individual cortical mesh, the lead field matrix was calculated and an adaptive spatial filter was applied separately for each frequency band (regularization: $\lambda=5 \%$ ). Power was computed for each source position. The coherency coefficient, which quantifies phase synchrony between 2 signals, was estimated between all pairs of sources ( $\mathrm{n}$ $=2,338)$. We then investigated the absolute imaginary part of coherency to reduce contributions to the connectivity estimate, which are due to potential field spread. ${ }^{21}$ In sum, a symmetrical, weighted, and undirected individual functional connectivity matrix was constructed for each frequency band. We averaged the weights of each vertex to estimate the overall connection strength of a vertex. To obtain an overall indicator of metrics, we also averaged connectivity and power across all vertices, yielding one global value per participant.

\section{Statistical Analysis of Imaging Metrics}

Group differences in power and connectivity were assessed using Permutation Analysis of Linear Models (fsl.fmrib.ox. ac.uk/fsl/fslwiki/PALM), a nonparametric statistical tool. In order to allow permutation inference in presence of possible dependence structures among related participants, we applied multilevel block permutation. ${ }^{22}$ Blocks of exchangeable units were defined and shuffled as a whole (families and single unrelated participants). Observations within a block were rearranged among themselves (observations within families). We carried out single $t$ contrasts instead of an overall $F$ test, which would have only allowed limited exchangeability of the data given related family structures (patients/siblings) and unrelated controls. Based on previous work, ${ }^{15,16}$ we hypothesized increased network levels in patients with GGE compared with controls and thus ran one-sided comparisons (controls $<$ siblings, siblings $<$ patients, controls < patients). Groups were contrasted vertexbased and on a global level, respectively, and for each frequency band separately. For each comparison, a general linear model was fit for every permutation, with imaging metrics as dependent variables. Group association and age constituted the predictors. Sex was initially included as additional predictor in the model but did not change any of the main results and was not further considered in the analyses. The data were permuted 5,000 times. An estimate of the empirical distribution of the $t$ statistics under the null hypothesis was constructed, from which the $p$ values were generated. In the vertex-based analysis, we corrected for multiple comparisons on cluster level using threshold-free cluster enhancement. ${ }^{23} p$ Values were familywise error corrected (FWE) within each group contrast and indicated as $-\log 10 p$ with a significance threshold of $1.3(p<0.05)$. Effect sizes (Cohen $d$ ) for vertex-based and global group comparisons were derived from the $t$ values of the linear models. $d$ Is therefore adjusted for age effects. An effect size of $d=0.2$ is considered to be small, $d=0.5$ intermediate, and $d=0.8$ large. $^{24}$

\section{Heritability of Imaging Patterns}

We explored the extent to which imaging phenotypes are heritable and quantified this using intraclass correlation (ICC) through linear mixed-effects modeling. ${ }^{25}$ ICC values were estimated based on the random effect components of a mixed model, which allows the incorporation of confounding effects. Here, a mixed model was constructed for power and connectivity, respectively, as dependent variables, family membership as random effect alongside group (patients vs siblings), and age as subject-level covariate (fixed-effect). $\operatorname{ICC}(1,1)$ was computed based on the variances of the random effect (family) and the total random effect variance (family and residual variance) ${ }^{25}$ using $\mathrm{R}$ (nlme package; CRAN.R-project.org/package $=$ nlme) and restricted maximum-likelihood estimation. ICC ranges from 0 to 1 , where an ICC close to 1 indicates correlated connectivity and power levels for patient-sibling pairs in a family. A low ICC means that family affiliation is not relevant and thus genetic contribution is unlikely. In total, 14 GGE families contributed to the ICC estimations. We performed a regional resampling of the vertex-level metrics using the Desikan-Killiany atlas ${ }^{26}$ to improve the signal-to-noise ratio (SNR) and calculated ICCs for 80 anatomically defined cortical and subcortical regions. Finally, we investigated whether heritability estimates of imaging metrics are particularly high in brain areas where patients show stronger differences from controls. To this end, effect sizes (Cohen $d$ ) from the vertex-wise group comparisons were averaged for each anatomical region and related to the ICC maps using Spearman rank correlation for each frequency band and metric. Higher positive correlations of effect sizes and ICC values imply genetic contribution to diseaserelated patterns in GGE.

\section{Data Availability}

All relevant data including power and connectivity results and ICC estimations are available from the corresponding author upon request. Raw imaging data are not publicly available due to data protection regulations. 


\section{Results}

\section{Participants}

Twenty-eight patients with GGE, 21 siblings, and 50 controls underwent resting-state measurements. We excluded participants due to technical problems during the acquisition $(\mathrm{n}=$ $5)$, movement artifacts $(n=4)$, or sleep $(n=2)$, leaving datasets of 25 patients, 18 siblings (related to 15 patients), and 45 controls for further analysis. Raw data from 6 patients were also used in a previous study. ${ }^{15}$ Anatomical MRI scans were visually rated as normal in all controls, siblings, and in 22 patients. Three patients had nonspecific findings (2 uncomplicated cysts, a single unspecific white matter lesion). Demographics and clinical details are described in the table. Family membership and GGE syndromes are indicated in figures $1 \mathrm{~A}$ and $2 \mathrm{~A}$. The groups were comparable for age (analysis of variance, $p=0.95$ ) and $\operatorname{sex}\left(\chi^{2}, p=0.84\right)$.

\section{Connectivity Analysis}

Compared with controls, patients with GGE showed increased functional connectivity in most of the frequency bands studied. Global connectivity (figure $3 \mathrm{~A}$ ) was higher in the $\theta\left(t_{67}=2.17, p=0.011, d=0.54\right), \alpha\left(t_{67}=2.36, p=0.016\right.$, $d=0.59), \beta 1\left(t_{67}=3.35, p=0.0004, d=0.84\right), \beta 2\left(t_{67}=2.40, p\right.$ $=0.023, d=0.60)$, and $\gamma$ band $\left(t_{67}=3.17, p=0.008, d=0.79\right)$, but not in $\delta\left(t_{67}=0.88, p=0.152, d=0.22\right)$. Vertex-based comparisons showed widespread bilateral increases across the frequency spectrum (figure 3B). Strongest effects were observed in the $\beta 1$ frequency band with a focus on lefthemispheric temporal, frontal, central, and parietal regions. Mesio-frontal regions were also pronounced in $\alpha, \beta 2$, and $\gamma$ frequency bands and postcentral regions mainly in the $\theta$ band. Connectivity of siblings statistically fell between patients and healthy controls (figure 3A). Global connectivity of patients was higher than in siblings for $a\left(t_{40}=1.67, p=0.047, d=\right.$ $0.52), \beta 2\left(t_{40}=1.98, p=0.027, d=0.61\right)$, and $\gamma\left(t_{40}=1.89, p=\right.$ $0.044, d=0.59)$, but there were no significant differences in the remaining frequency bands $\left(\delta: t_{40}=1.38, p=0.098, d=\right.$ $0.43 ; \theta: t_{40}=1.19, p=0.247, d=0.37 ; \beta 1: t_{40}=0.90, p=0.205$, $d=0.28)$. Siblings did not significantly differ from controls $(\delta$ : $t_{60}=-0.8, p=0.444, d=-0.21 ; \theta: t_{60}=0.49, p=0.223, d=$ $0.14 ; \alpha: t_{60}=0.45, p=0.267, d=0.13 ; \beta 1: t_{60}=1.59, p=0.101$, $d=0.44 ; \beta 2: t_{60}=0.46, p=0.250, d=0.13 ; \gamma: t_{60}=0.749, p=$ $0.520, d=0.21$ ). On a vertex level (figure $3 \mathrm{C}$ ) and after correction for multiple comparisons, siblings differed from patients with GGE in $\alpha$ and $\beta 2$ frequency bands ( $p_{\mathrm{FWE}}<$ $0.05)$, but not from controls $\left(p_{\mathrm{FWE}}>0.05\right)$.

\section{Power Analysis}

Patients with GGE had higher power than controls in all frequency bands studied, in the global (figure $4 \mathrm{~A} ; \delta: t_{67}=$ $3.15, p=0.002, d=0.79 ; \theta: t_{67}=3.73, p=0.0002, d=0.93$;

Table Study Population

\begin{tabular}{|c|c|c|c|}
\hline & Patients & Siblings & Controls \\
\hline Total & 25 & 18 & 45 \\
\hline Female & $16(64)$ & $10(55)$ & $28(62)$ \\
\hline Age, y & $25(22-37)$ & $26(22-42)$ & $25(23-35)$ \\
\hline Positive family history of epilepsy/seizures & $12(48)$ & $7(39)^{a}$ & $0(0)$ \\
\hline GSWD during MEG recordings & $9(36)$ & $0(0)$ & $0(0)$ \\
\hline GSWD in routine/long-term EEG & $22(88)$ & - & - \\
\hline Seizure free $>12$ months & $16(64)$ & - & - \\
\hline Drugs at measurement & $1.2(0-3)$ & - & - \\
\hline Epilepsy syndrome & & - & - \\
\hline CAE & $5(20)$ & - & - \\
\hline JAE & $6(24)$ & - & - \\
\hline JME & $5(20)$ & - & - \\
\hline GTCS & $4(16)$ & - & - \\
\hline GGE & $5(20)$ & - & - \\
\hline Age at onset, y & $15(10-17)$ & - & - \\
\hline Disease duration, y & $17(8-24)$ & - & - \\
\hline
\end{tabular}

Abbreviations: CAE = childhood absence epilepsy; GGE = genetic generalized epilepsy (unclassified); GSWD = generalized spike-wave discharges; GTCS = generalized tonic-clonic seizures; JAE = juvenile absence epilepsy; JME = juvenile myoclonic epilepsy; MEG = magnetoencephalography.

Values are $n(\%)$, median (interquartile range), or mean (range).

a Positive family history beyond the related index patient with GGE. 
A. Global connectivity within families and GGE syndromes

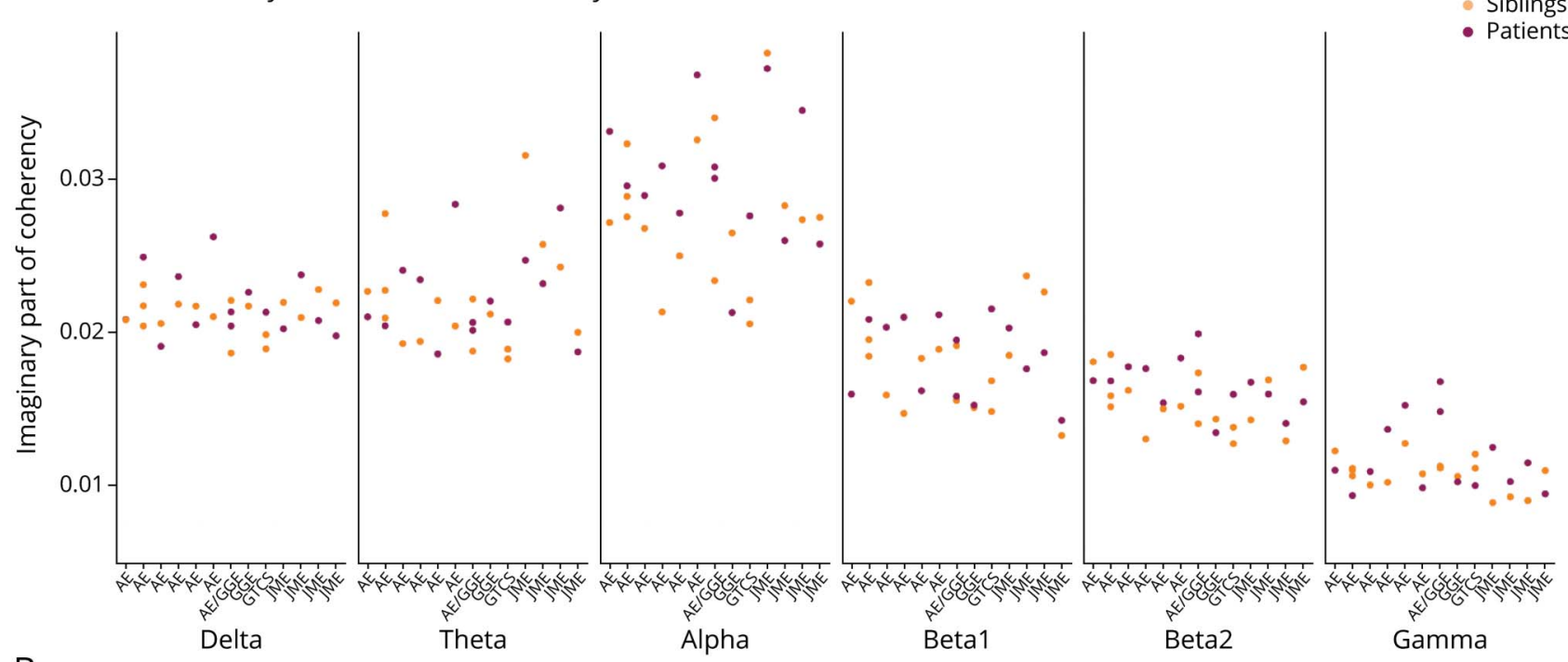

B. ICC for connectivity differences (GGE > controls)

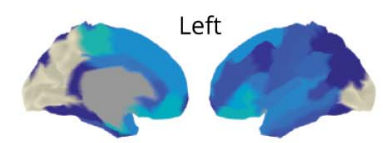

Beta1 $16 \pm 4$
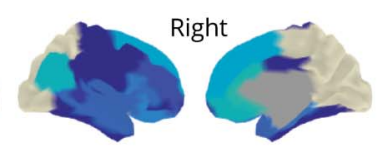

Left

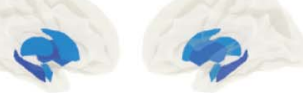

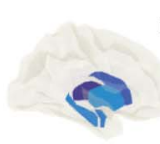

Right

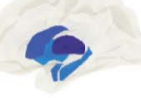

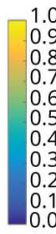

ICC

(A) Individual global connectivity values of patients with GGE and siblings are plotted with regard to their family membership (columns of data within frequency bands). Data of patients with GGE without a corresponding sibling are not shown. GGE syndromes of patients in each family are indicated on the xaxis. Childhood absence epilepsy and juvenile absence epilepsy are referred to absence epilepsies (AE). (B) Color-coded heritability estimates (intraclass correlation [ICC] values) per region based on the Desikan-Kiliany atlas ${ }^{26}$ with small to large group-level differences between patients with GGE and controls (Cohen $d>0.2$ ). Only ICC maps with a positive and significant correlation with averaged effect sizes are shown ( $p<0.05$ ). The color coding indicates the strength of ICC values in those regions for connectivity differences. ICC estimates were derived from random effect components of mixed models for each region, taking group and age effects into account. A large ICC indicates correlated imaging patterns for patient-sibling pairs in a family ( $\mathrm{n}=14$ ) and thus heritability of the metrics. Cortical regions are displayed in the left column and subcortical regions are shown separately in the right column of the plot. GTCS = generalized tonic-clonic seizures; JME = juvenile myoclonic epilepsy.

$\alpha: t_{67}=4.22, p=0.0002, d=1.05 ; \beta 1: t_{67}=5.44, p=0.0002$, $d=1.36 ; \beta 2: t_{67}=4.24, p=0.0002, d=1.06 ; \gamma: t_{67}=3.60, p$ $=0.0006, d=0.90)$ and vertex-based analysis $\left(p_{\mathrm{FWE}}<0.05\right.$; figure 4B). Differences were focused on occipital-parietal and temporal regions. Also, hippocampal $(\alpha, \beta 1, \beta 2)$ and subcortical structures such as thalamus and putamen $(\beta 1)$ showed higher power. Patients also exhibited higher global power than siblings (figure $4 \mathrm{~A})$ in $\alpha\left(t_{40}=2.40, p=0.027\right.$, $d=0.74), \beta 1\left(t_{40}=1.34, p=0.024, d=0.41\right)$, and $\gamma\left(t_{40}=\right.$ $1.74, p=0.048, d=0.54)$, but not in $\delta\left(t_{40}=1.96, p=0.123\right.$, $d=0.61), \theta\left(t_{40}=1.60, p=0.189, d=0.50\right), \beta 2\left(t_{40}=0.68, p\right.$ $=0.096, d=0.21)$ frequency bands. In the vertex-based comparison, patients with GGE had higher power than siblings in all frequency bands but $\theta$ (figure 4C), mainly in occipital regions. Global power in siblings was higher than in controls (figure 4A) in $\beta 1\left(t_{60}=2.53, p=0.044, d=0.71\right.$ ) and $\beta 2$ bands $\left(t_{60}=2.62, p=0.044, d=0.73\right)$, but not in the remaining frequency bands $\left(\delta: t_{60}=0.16, p=0.191, d=\right.$ $0.04 ; \theta: t_{60}=1.28, p=0.108, d=0.36 ; \alpha: t_{60}=0.85, p=$ $\left.0.246, d=0.24 ; \gamma: t_{60}=1.02, p=0.193, d=0.29\right)$. Differences at vertices did not reach statistical significance $\left(p_{\mathrm{FWE}}>0.05\right)$.

\section{Heritability of Imaging Patterns}

ICC values, which represent heritability estimations of MEGderived patterns, strongly correlated with $\beta 1$ connectivity differences of patients against controls $\left(r_{s}=0.59, p=\right.$ $\left.1.88 \mathrm{e}^{-08}\right)$ and negatively to $\beta 2$ levels $\left(r_{s}=-0.39, p=4.23 \mathrm{e}^{-04}\right)$. The correlation of ICC and connectivity differences in the remaining frequency bands did not reach significance $\left(\delta: r_{s}=\right.$ -0.01, $p=0.900 ; \theta: r_{s}=-0.10, p=0.364 ; \alpha: r_{s}=0.14, p=$ $\left.0.223 ; \gamma: r_{s}=0.18, p=0.120\right)$. ICC values for power were significantly associated with GGE contrast maps in all frequency bands except $\alpha$ and $\gamma\left(\delta: r_{s}=0.60, p=8.6 \mathrm{e}^{-09} ; \theta: r_{s}=\right.$ $0.46, p=2.5 \mathrm{e}^{-05}$; $\alpha: r_{s}=0.18, p=0.109 ; \beta 1: r_{s}=0.59, p=$ $\left.1.47 \mathrm{e}^{-08} ; \beta 2: r_{s}=0.34, p=0.002 ; \gamma: r_{s}=0.15 p=0.17\right)$. In the following, we only report results for ICC maps with positive and significant correlations with the GGE phenotype $(p<$ $0.05)$, implying genetic contribution to disease-related patterns (figures $1 \mathrm{~B}$ and $2 \mathrm{~B}$ ). ICC values for connectivity in $\beta 1$ frequency band were highest in rostral and caudal anterior cingulate, orbitofrontal, paracentral, entorhinal, and inferior parietal regions $(\mathrm{ICC}>0.4)$ and lower in temporal regions and subcortical nuclei (ICC $>0.2$ ) figure $1 B)$. ICC estimates were generally higher for power than connectivity and peaked 
A. Global power within families and GGE syndromes

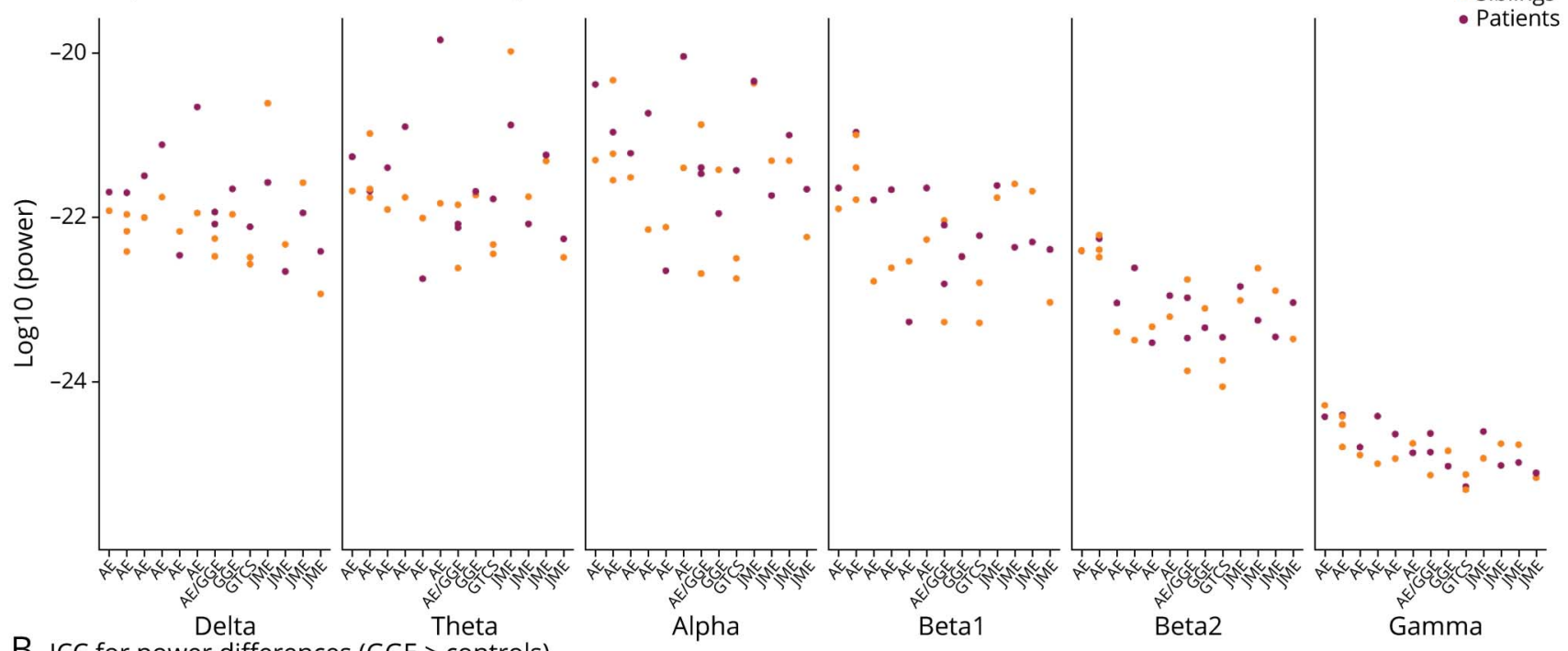

B. ICC for power differences (GGE > controls)

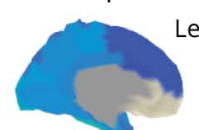

Delta $2 \pm 2 \mathrm{~Hz}$

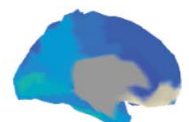

Theta $6 \pm 2 \mathrm{~Hz}$
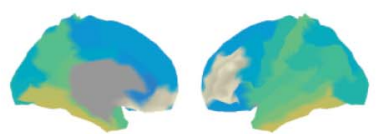

Beta1 $16 \pm 4 \mathrm{~Hz}$

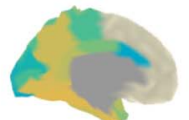

Beta2 $25 \pm 4 \mathrm{~Hz}$
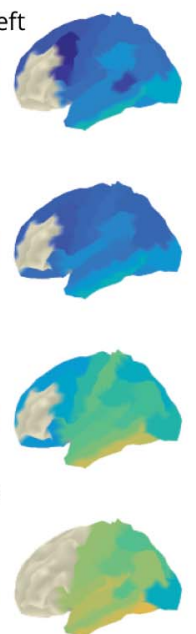
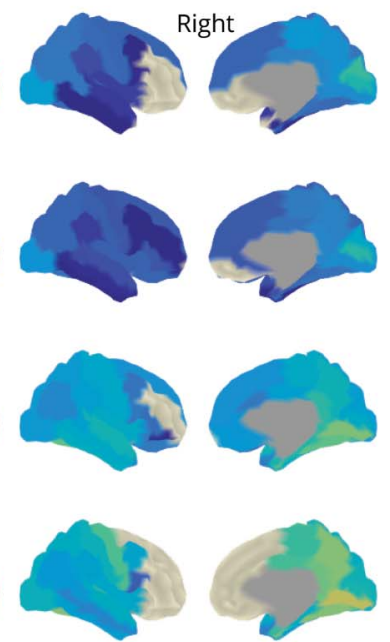

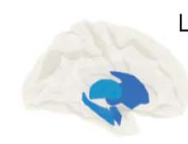

Left
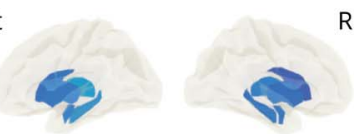

Right
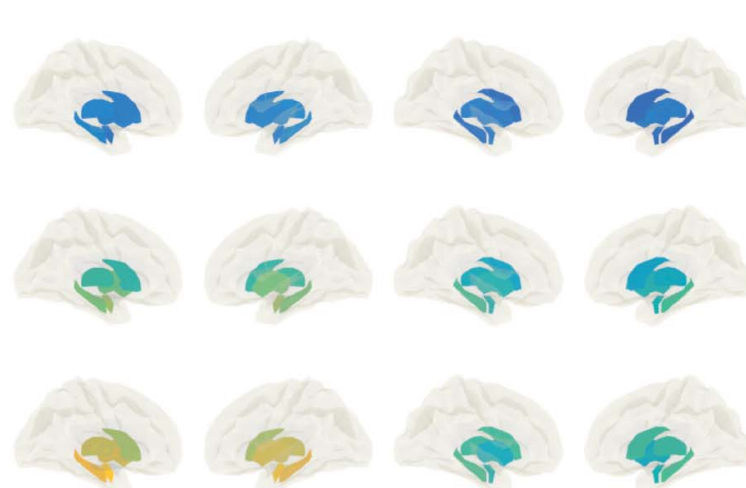

(A) Individual global power values of patients with GGE and siblings are plotted with regard to their family membership (columns of data within frequency bands). Data of patients with GGE without a corresponding sibling are not shown. GGE syndromes of patients in each family are indicated on the X-axis. Childhood absence epilepsy and juvenile absence epilepsy are referred to absence epilepsies (AE). For visualization purposes, power data were log10transformed. (B) Color-coded heritability estimates (intraclass correlation [ICC] values) per region based on the Desikan-Kiliany atlas ${ }^{26}$ with small to large group-level differences between patients with GGE and controls (Cohen $d>0.2$ ). Only ICC maps with a positive and significant correlation with averaged effect sizes are shown $(p<0.05)$. The color coding indicates the strength of ICC values in those regions for power differences. ICC estimates were derived from random effect components of mixed models for each region, taking group and age effects into account. A large ICC indicates correlated imaging patterns for patient-sibling pairs in a family $(n=14)$ and thus heritability of the metrics. Cortical regions are displayed in the left column and subcortical regions are shown separately in the right column of the plot. GTCS = generalized tonic-clonic seizures; JME = juvenile myoclonic epilepsy.

in $\beta$ frequency bands in temporal, subcortical, and parietal regions such as lingual gyrus and cuneus as well as postcentral gyrus (ICC > 0.5). ICC maps for $\delta$ and $\theta$ frequency bands showed similar patterns but generally lower ICC values (ICCs up to $\sim 0.5$ ) (figure $2 B$ ).

\section{Clinical Variables and Imaging Findings}

We carried out secondary analyses to evaluate the relation of clinical variables with brain oscillations. We investigated whether networks of patients with GGE with GSWD during the MEG recordings differ from patients without GSWD. Although trials containing GSWD ( \pm 10 seconds of data) were rejected in all analyses, patients with GSWD in the recording had higher connectivity in the $\delta$ frequency band (global: $t_{22}=$ 2.95, $p=0.004, d=1.26$; vertex-based: $p<0.05$; figure $5 \mathrm{~A}$ ). There was also a tendency of higher power across frequency bands in those patients, with significant differences for $\delta$ (global: $t_{22}=2.55, p=0.010, d=1.09$; vertex-based: $p<0.05$ ) and $\beta 1$ frequency bands (global: $t_{22}=2.23, p=0.019, d=0.96$; vertex-based: $p<0.05$; figure 5A). Mean global power and connectivity for patients without GSWD during the recording remained higher for patients than controls and never dropped below the mean levels of siblings (data not shown). Patients taking 2 or more drugs at the study date had lower 
A. Global connectivity all participants

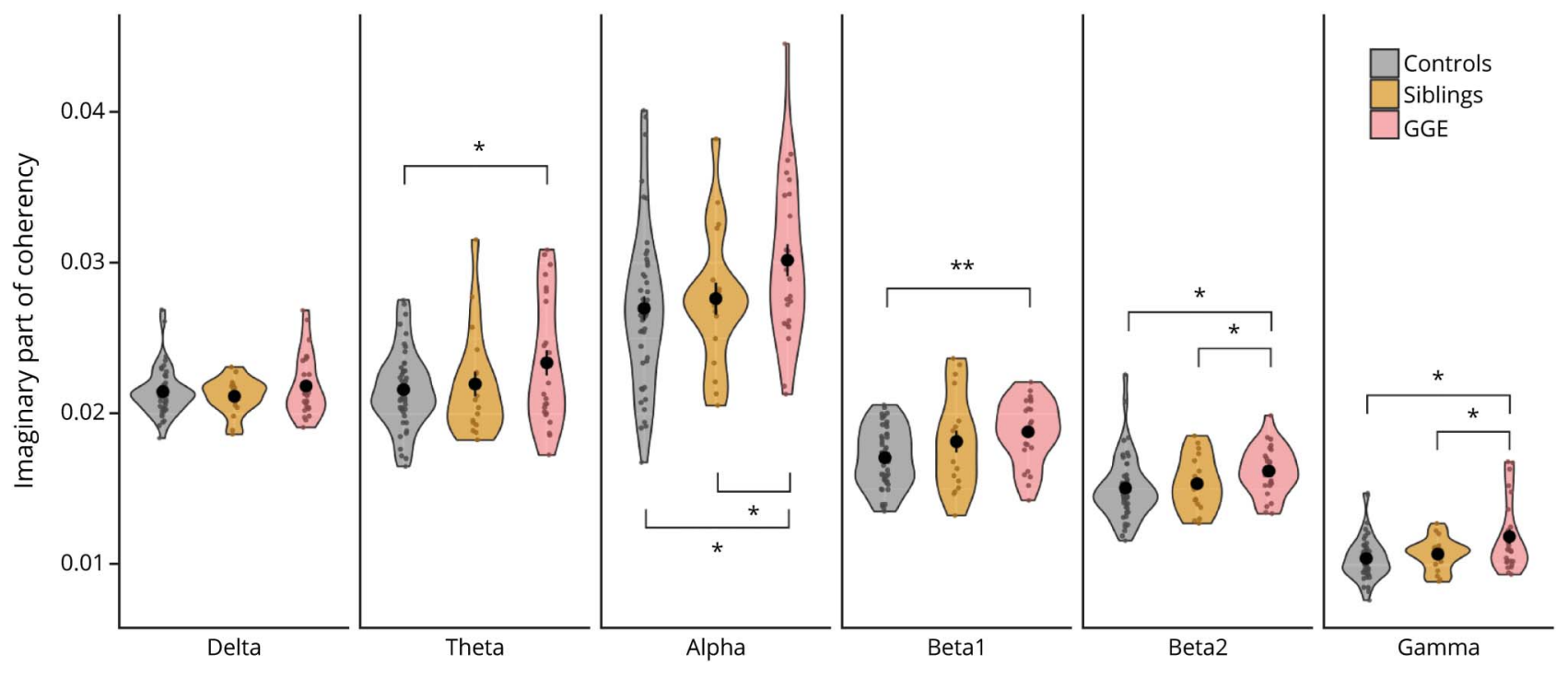

B. Vertex-connectivity GGE > controls

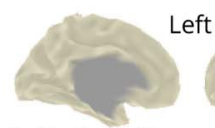

Delta $2 \pm 2 \mathrm{~Hz}$

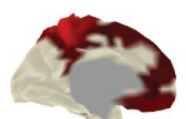

Theta $6 \pm 2 \mathrm{~Hz}$

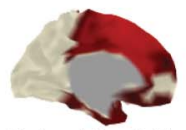

Alpha $10 \pm 2 \mathrm{~Hz}$

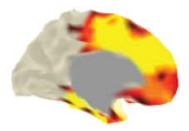

Beta1 $16 \pm 4 \mathrm{~Hz}$

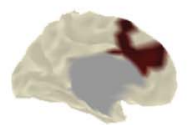

Beta2 $25 \pm 4 \mathrm{~Hz}$

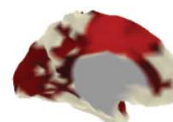

Gamma $40 \pm 8 \mathrm{~Hz}$
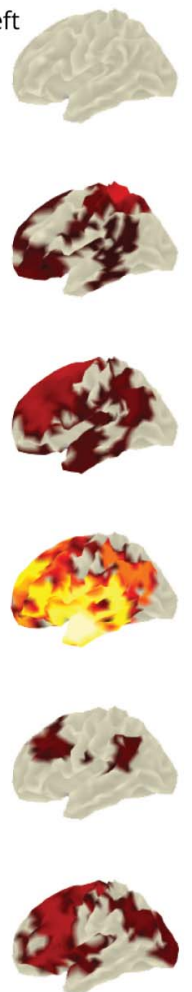
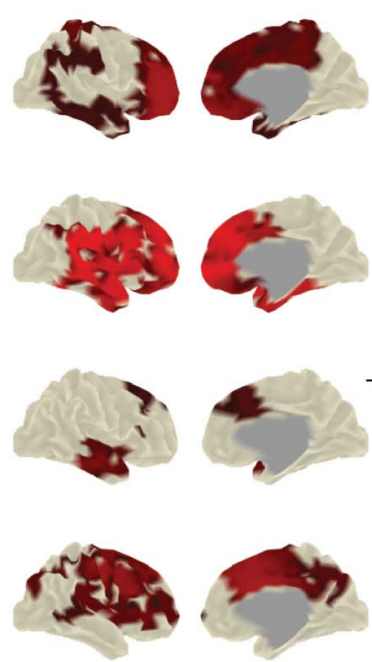

C. Vertex-connectivity GGE > siblings
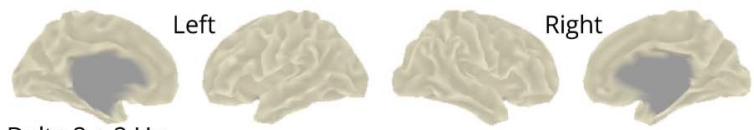

Delta $2 \pm 2 \mathrm{~Hz}$
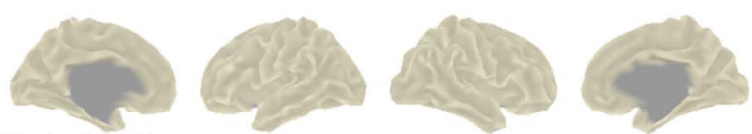

Theta $6 \pm 2 \mathrm{~Hz}$
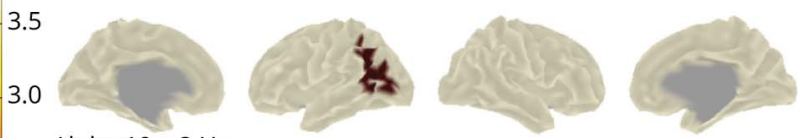

2.5 Alpha $10 \pm 2 \mathrm{~Hz}$
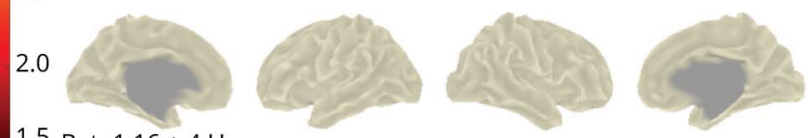

1.5 Beta1 $16 \pm 4 \mathrm{~Hz}$
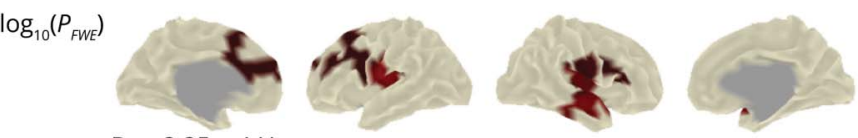

Beta2 $25 \pm 4 \mathrm{~Hz}$
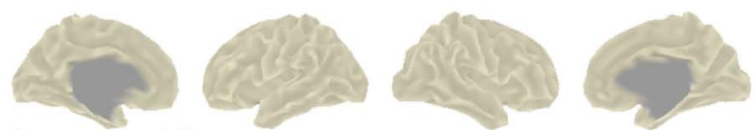

Gamma $40 \pm 8 \mathrm{~Hz}$

(A) Violin plots show individual data points, the density of the data, group means, and standard errors of the means for the global imaginary part of coherency in each frequency band (controls, $n=45$; siblings, $n=18$; patients with genetic generalized epilepsy [GGE], $n=25$ ). Asterisks denote statistical significance at * $p$ $<0.05$ and ${ }^{* *} p<0.001$ for permutation-based group comparisons. (B) The plot highlights vertices with significantly higher connectivity values in patients with GGE $(\mathrm{n}=25)$ than in controls $(\mathrm{n}=45)$ and $(C)$ higher connectivity values in patients with GGE $(\mathrm{n}=25)$ than in siblings $(\mathrm{n}=18)$. The color scale indicates -log10 $p$ with a cutoff of 1.3 (corresponding to $p<0.05$, familywise error corrected). In all analyses, age was included as covariate of no interest.

connectivity in $\beta 1$ frequency band than patients taking less than 2 drugs (global: $t_{21}=-2.04, p=0.029, d=-0.98$; vertexbased: $p<0.05$, figure 5C). The effect was stronger when accounting for the presence of GSWD during MEG recordings. There were no differences for power in any of the frequency bands studied (all $p>0.05$ ). Other clinical factors such 
A. Global power all participants

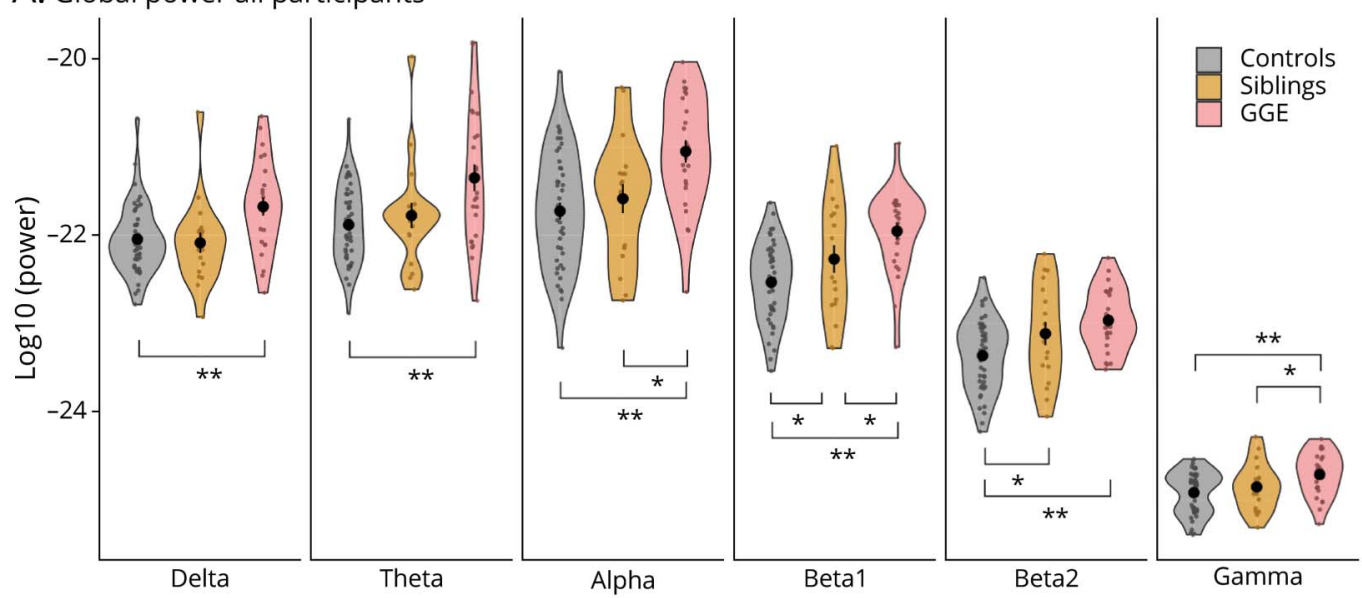

B. Vertex-power GGE > controls

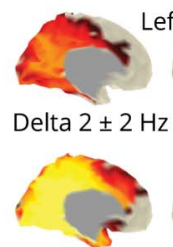

Theta $6 \pm 2 \mathrm{~Hz}$

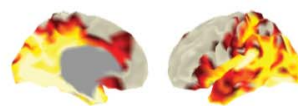

Alpha $10 \pm 2 \mathrm{~Hz}$

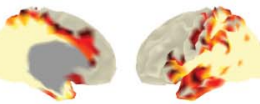

Beta1 $16 \pm 4 \mathrm{~Hz}$

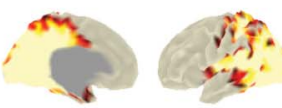

Beta2 $25 \pm 4 \mathrm{~Hz}$

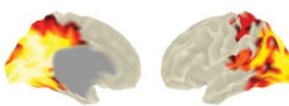

Gamma $40 \pm 8 \mathrm{~Hz}$

Left

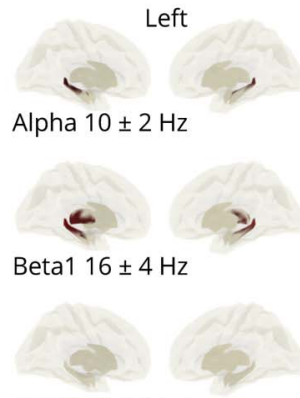

Beta2 $25 \pm 4 \mathrm{~Hz}$
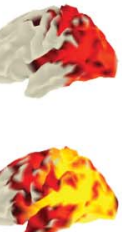
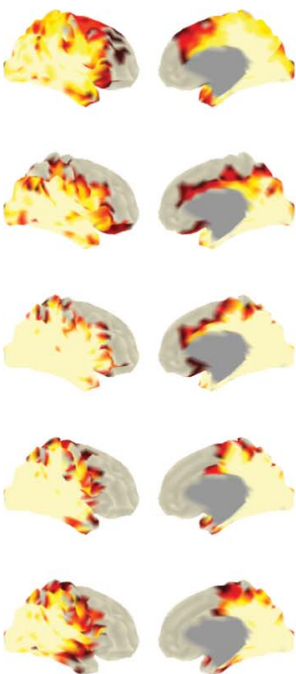

Right

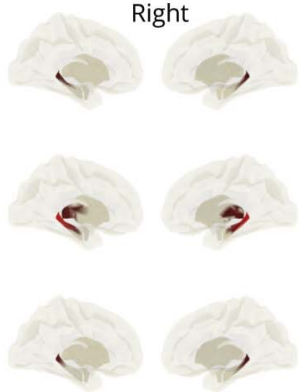

(A) Violin plots show individual data points, the density of the data, group means, and standard errors of the means for global power in each frequency band (controls, $\mathrm{n}=45$; siblings, $\mathrm{n}=18$; patients with genetic generalized epilepsy [GGE], $\mathrm{n}=25$ ). Asterisks denote statistical significance at * $p<0.05$ and ** $p<0.001$ for permutation-based group comparisons. For visualization purposes, power data were log10-transformed. (B) The plot highlights cortical and subcortical vertices with significantly higher power values in patients with GGE $(n=25)$ than in controls $(n=45)$. (C) The plot shows vertices with significantly higher power values in patients with GGE $(n=25)$ than in siblings $(n=18)$. The color scale indicates $-\log 10 p$ with a cutoff of 1.3 (corresponding to $p<0.05$, familywise error corrected). In all analyses, age was included as covariate of no interest. 
A. Vertex-connectivity GSWD > no GSWD
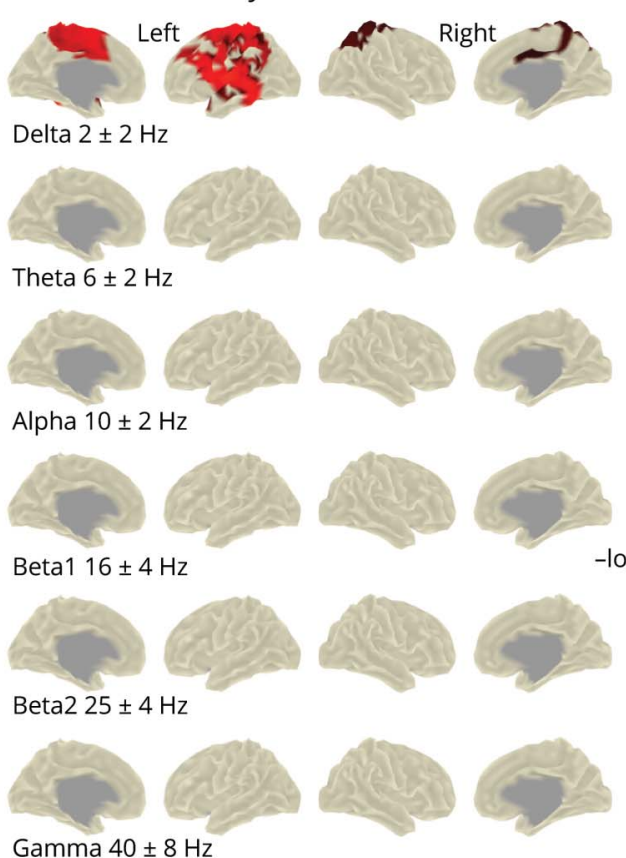

Gamma $40 \pm 8 \mathrm{~Hz}$
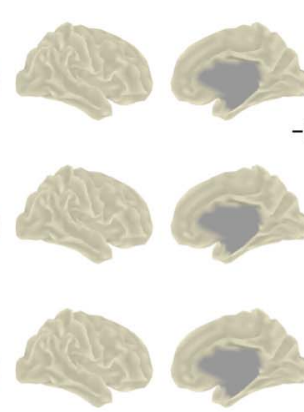
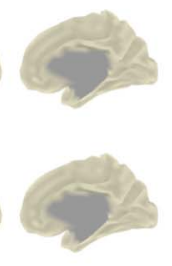

\section{$-\log _{10}\left(P_{\text {FWE }}\right)$ Beta1 $16 \pm 4 \mathrm{~Hz}$}
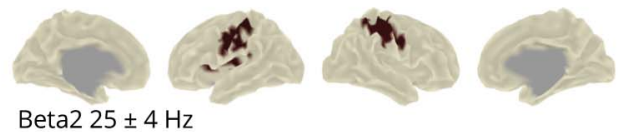

Beta2 $25 \pm 4 \mathrm{~Hz}$
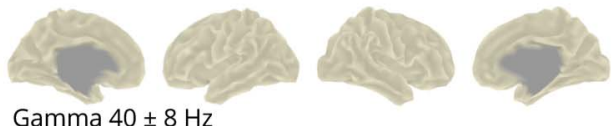

C. Vertex-connectivity 0-1 AEDS > 2+ AEDS

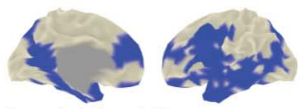

Beta1 $16 \pm 4 \mathrm{~Hz}$

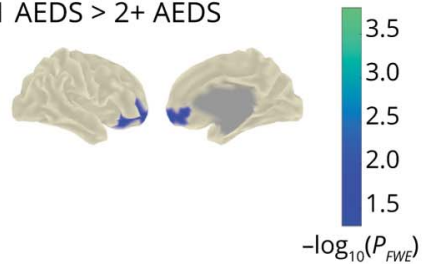

3.5

3.0

2.5

5$$
\text { ) }
$$

Vertex plots highlight cortical vertices with higher (A) connectivity and (B) power values in patients with genetic generalized epilepsy (GGE) with generalized spike-wave discharges (GSWD) $(n=9)$ than patients without GSWD $(n=16)$ during the magnetoencephalography recordings. These effects were present after exclusion of trials containing GSWD \pm 10 seconds of data and corrected for age effects. (C) The plot shows significantly lower connectivity in patients with GGE taking 2 or more antiepileptic drugs $(n=6)$ than patients taking fewer than 2 drugs $(n=19)$ at the study date. Age and presence of GSWD was included as covariate of no interest. Color scales indicate $-\log 10 p$ with a cutoff of 1.3 (corresponding to $p<0.05$, familywise error corrected).

as age at onset, disease duration, or seizure control were not associated with global and vertex-based connectivity or power (all $p>0.05)$.

\section{Discussion}

We assessed power and phase-based connectivity during rest in patients with GGE and their healthy siblings to evaluate endophenotypic potential of electrophysiologic metrics. Patients with GGE showed bilateral, widespread, and highly increased power and connectivity compared with controls. Asymptomatic siblings presented with intermediate levels between patients and controls, particularly in $\beta$ frequencies, suggesting genetic background as major driver for those patterns.

We expanded and replicated previous work of ours using MEG data of mixed GGE cohorts, ${ }^{15,16}$ confirming strong interictal network differences in GGE as quantified with the investigated imaging metrics. Both global and local measures have been reliable in source-space analyses using the same processing pipeline. ${ }^{20}$ Increased power in GGE is a previously reported finding, ${ }^{27,28}$ but $\mathrm{M} / \mathrm{EEG}$ connectivity studies on source level are scarce. ${ }^{15,16}$ High $\beta$ band connectivity in GGE was the most consistent finding across the studies, followed by increases in $\theta$, $\alpha$, and $\gamma$ bands. Epileptic seizures commonly involve pathologic synchronization. ${ }^{29}$ Also during the interictal state, patients have had higher liability to synchronize. ${ }^{30,31}$

Increased network connectivity and power was also observed in siblings without active epilepsy. ${ }^{6}$ Intraclass correlations substantiate heritability in regions, where patients with GGE strongly differed from controls, specifically for $\beta 1$ band connectivity and both $\beta 1$ and $\beta 2$ power. In $\delta$ and $\theta$ power, heritability estimates corresponded to the phenotype of patients, but effect sizes and ICC values were comparably low. Increased $\beta 2$ connectivity patterns in patients compared with controls were less concordant within families. 
Elevated MEG connectivity was mostly heritable in anterior cingulum, orbitofrontal, and superior frontal regions. In GGE, direct evidence for a genetically determined functional dysregulation in frontal cortex is limited, but has been documented for prefrontal and cingulate morphology. ${ }^{10}$ Altered structural network integration of the frontal cortex ${ }^{10}$ potentially leads to cognitive impairments in patients with JME and similarly in their healthy siblings. ${ }^{7-10}$ Furthermore, network power and connectivity was heritable in central brain regions. Hyperactivations in motor systems have been suggested as endophenotypes of JME during cognitive fMRI, ${ }^{13,14}$ but also for a mixed GGE cohort during resting-state. ${ }^{30}$ We complement these findings by studying much faster neuronal oscillations using MEG and without vascular confounds. Our results point to more globally increased network levels in patients with GGE and siblings, irrespective of a task involved. This includes increased power patterns in mesiotemporal cortices and in subcortical structures with a strong genetic substrate. ICC estimates reached levels of up to 0.85 , particularly in $\beta$ frequencies, indicating that familial and, thus, likely genetic factors explain the majority of the observed variance. Thalamocortical circuits are critical for $\mathrm{GGE}^{32}$ and structural alterations in the thalamus ${ }^{33,34}$ have been linked to subcortico-cortico hub organization in GGE. ${ }^{33}$ Using MEG, it is debatable whether signals of deep brain structures can be captured. Yet recent work has used simultaneous intracerebral and MEG recordings in patients with epilepsy and demonstrated detectability of signals generated in mesial temporal lobe structures as well as thalamic activity at the surface. ${ }^{35}$ In line with our results, mesiotemporal task-based activations cosegregated in patients with JME and their healthy siblings, along with changes in hippocampal morphology. ${ }^{11}$ The role of increased occipital power in our GGE cohort is less clear. Low-density EEG data ${ }^{28}$ and microstructural alterations in fronto-occipital white matter association tracts ${ }^{36}$ point to the involvement of occipital areas in GGE. In our study, patients and siblings mostly differed in occipital power, suggesting that this finding may not be due to genetic factors but to other unknown disease-related effects. Overall, we consolidate findings of earlier GGE-sibling studies using (f)MRI and add evidence for resting-state trait heritability in extended brain networks at higher temporal resolution.

Electrophysiologic studies in twins suggest strong genetic influence on brain oscillations relative to environmental factors, particularly for wideband power $\left(h^{2} \sim 0.5-0.8\right) .{ }^{18,37}$ Heritability for connectivity on the cortical source level has been lower than for power and highest in $\alpha$ and $\beta$ frequencies $(10 \%-20 \%) .{ }^{19}$ Lower reproducibility of connectivity metrics may, at least partly, explain a lower heritability estimate compared to power. Similarly, connectivity in $\alpha$ and $\beta$ bands has been more repeatable than in other frequency bands. ${ }^{20}$ Network alterations characterizing GGE were captured in brain rhythms of different frequencies, but imaging phenotypes of siblings and patients with GGE most strongly correlated in $\beta$ frequencies. $\beta$ band oscillations are classically related to sensory and motor processing ${ }^{38}$ as well as longdistance synchronization. ${ }^{39}$ GABAergic processes are presumably involved in the generation of MEG $\beta$ oscillations as shown with endogenous GABA concentrations in humans. ${ }^{40}$ GABAA receptor gene variants constitute GGE disease risk ${ }^{5,41}$ and one of those candidates, the GABRA2 gene, has been linked to EEG $\beta$ band activity. ${ }^{42,43}$ Moreover, recently discovered genetic markers in GGE have been enriched in the frontal cortex, specifically in the dorso-lateral prefrontal cortex. ${ }^{5}$ Genetic signals have further converged on the inferior temporal lobe, angular gyrus, cingulum, and subcortical tissue, but less strongly. ${ }^{5}$ Our ICC maps show substantial spatial correspondence to those findings, emphasizing the functional relevance of imaging resting-state markers as investigated in our study. Previously, oscillatory activity has been successfully used to detect psychiatric liability genes ${ }^{43}$ and EEG coherence has served as an endophenotype for alcohol use disorders. ${ }^{44}$ Yet the functional role of spectral perturbations is not fully understood. Here, we can only speculate about molecular changes occurring within specific networks in the brain, such as an excitation-inhibition imbalance, as a putative key factor in epilepsy. Through coupling mechanisms, the coordination of neuronal spike timing might be affected across networks, in sum leading to increased oscillatory amplitudes. ${ }^{45}$

Disease duration and age at onset did not correlate with the imaging patterns in our GGE cohort. These clinical variables do not necessarily describe disease severity and the lack of a significant association with the imaging findings may support the notion of a genetic imaging trait. Patients showing epileptic discharges during the MEG recording had higher $\delta$ connectivity and $\delta$ and $\beta$ power increases at rest (after careful exclusion of segments with GSWD \pm 10 seconds of data). These patterns had a spatial profile with a temporal and central focus. GSWD typically have a frequency around $3 \mathrm{~Hz}$ and it is possible that we captured network dynamics around the onset or offset, evolving during a considerable time span. ${ }^{31}$ Only 9 patients in our study showed GSWD and at least 5 of them had experienced seizures within the past year. However, seizure control was not associated with significantly different network patterns in our cohort and might not be a sensitive marker in a rather wellcontrolled cohort. Similarly, persistent GSWD do not necessarily have an effect on long-term seizure prognosis. ${ }^{46}$ Moreover, patients with higher medication load had lower connectivity in the $\beta 1$ frequency band. Because only 6 patients received more than 2 antiepileptic agents at the time of the study, we can only speculate about a network downregulation through antiepileptic treatment. Normalizing effects of antiepileptic drugs on background synchronization have been demonstrated before. ${ }^{47}$ Further investigations are needed to confirm our exploratory results and study drug-specific effects.

This study has limitations. We found alterations for both power and imaginary part of coherency, a phase-based metric. Power and phase characterize different aspects of neural signals; however, both physiologic and nonphysiologic coupling between those characteristics has been noted. ${ }^{45}$ In particular, high values in phase-based connectivity require a temporally stable phase relationship of signals, which depends on the 
SNR and eventually on signal power. In our study, the topography for GGE power differences had a posterior focus and was distinct from connectivity maps with a more frontal emphasis. Recent work suggests that spontaneous MEG networks can be decoupled into anterior and posterior states, both connected to the posterior cingulate cortex, likely reflecting functional specialization. ${ }^{48}$ Given the different effects of power and connectivity on the MEG signal topography, it is very likely that both measures captured independent features. Furthermore, we cannot assess the mechanisms of genetic control over oscillatory markers. Methodologic aspects may also affect heritability estimates. For example, higher sensitivity of connectivity measures to noise might be a reason for generally lower effect sizes than for power and weaker ICC values in our study. Accordingly, higher statistical power is needed for significant connectivity differences between siblings and controls or patients, given the intermediate levels of the siblings. The relatively small number of $14 \mathrm{fam}$ ilies that were available for the study also limits the precise estimation of the ICCs. Finally, we cannot discern specific effects for the GGE subsyndromes due to the small sample size. Yet multiple GGE subtypes occur within the same families ${ }^{49}$ and overlap of genetic risk factors has been suggested. ${ }^{5}$ Network phenotypes as assessed in our study could reflect shared pathophysiologic features across the syndromes, such as the occurrence of GSWD. Interestingly, GSWD have been more frequently observed in unaffected first-degree relatives of patients with GGE than in the general population, ${ }^{50}$ which again points to genetic contributions to network function in GGE.

We propose that increased interictal MEG power and connectivity in frontocentral and temporo-parietal cortical regions are a hallmark of GGE. These network features are likely driven by genetic factors and not by the presence or absence of the active disease or clinical confounds. Siblings without epilepsy had similarly increased network levels during rest, predominantly in $\beta$ frequencies. We show that power and phase-based connectivity are heritable and may serve as markers to link imaging with genetics in epilepsy.

\section{Acknowledgment}

The authors thank the patients, their families, and controls for taking part in this study; Dr. Gang Chen (Scientific and Statistical Computing Core, National Institute of Mental Health, Bethesda, MD) for discussions on statistics; Dr. Florian Klinker (Clinic of Clinical Neurophysiology, University Medical Center Göttingen) for reviewing epileptic discharges in MEG recordings; Dr. Silke Klamer-Ethofer (Department of Neurology and Epileptology, Hertie Institute of Clinical Brain Research, University of Tübingen, Germany) and Silvia Vannoni (Department of Neurology and Epileptology, Hertie Institute of Clinical Brain Research) for assistance in data acquisition; and Prof. Dr. Yvonne Weber (Department of Neurology and Epileptology, Hertie Institute of Clinical Brain Research) for providing clinical information.

\section{Study Funding}

This work was supported by the DFG (Deutsche Forschungsgemeinschaft, grant number FO 750/5-1 to N.K.F.).

\section{Disclosure}

N.K. Focke received speaker bureau and consultancy fees from Bial, UCB, Eisai, and EGI/Phillips, all unrelated to the present work. H. L. received speaker bureau and consultancy fees from Arvelle, Bial, Biomarin, Eisai and UCB, all unrelated to the present work. C. Stier, A. Elshahabi, Y. Li Hegner, R. Kotikalapudi, J. Marquetand, and C. Braun report no disclosures. Go to Neurology.org/N for full disclosures.

\section{Publication History}

Received by Neurology October 19, 2020. Accepted in final form April 7, 2021.

\section{Appendix Authors}

\begin{tabular}{lll}
\hline Name & Location & Contribution \\
\hline Christina & Clinic of Clinical & $\begin{array}{l}\text { Designed and } \\
\text { Stier, MSc }\end{array}$ \\
& $\begin{array}{l}\text { Neurophysiology, University } \\
\text { Medical Center Göttingen; } \\
\text { and Department of }\end{array}$ & $\begin{array}{l}\text { major role in the acquisition } \\
\text { of data, analyzed the data, }\end{array}$ \\
& $\begin{array}{l}\text { Neurology and Epileptology, } \\
\text { Hertie Institute of Clinical } \\
\text { Brain Research, University } \\
\text { of Tübingen, Germany }\end{array}$ & $\begin{array}{l}\text { analyses, interpreted the } \\
\text { results, drafted the } \\
\text { manuscript }\end{array}$ \\
& & \\
&
\end{tabular}

Adham Department of Neurology and Major role in the acquisition
Elshahabi, Epileptology, Hertie Institute of data, revised the MD, MSc of Clinical Brain Research, manuscript for intellectual University of Tübingen, content

Germany; and Department of Neurology, University Hospital Zurich, Switzerland

\begin{tabular}{lll}
\hline $\begin{array}{l}\text { Yiwen Li } \\
\text { Hegner, MD, } \\
\text { PhD }\end{array}$ & $\begin{array}{l}\text { Department of Neurology } \\
\text { and Epileptology, Hertie } \\
\text { Institute of Clinical Brain } \\
\text { Research, University of } \\
\text { Tübingen, Germany }\end{array}$ & $\begin{array}{l}\text { Provided critical input on } \\
\text { the methods, revised the } \\
\text { manuscript for intellectual } \\
\text { content }\end{array}$ \\
\hline $\begin{array}{l}\text { Raviteja } \\
\text { Kotikalapudi, } \\
\text { PhD }\end{array}$ & $\begin{array}{l}\text { Clinic of Clinical } \\
\text { Neurophysiology, University } \\
\text { Medical Center Göttingen; } \\
\text { Department of Neurology } \\
\text { and Epileptology, Hertie } \\
\text { Institute of Clinical Brain }\end{array}$ & $\begin{array}{l}\text { Major revised the } \\
\text { manuscript for intellectual } \\
\text { content }\end{array}$ \\
& $\begin{array}{l}\text { Research, University of } \\
\text { Tübingen, Germany; and } \\
\text { Institute of Psychology, }\end{array}$ & \\
& University of Bern, \\
& Switzerland
\end{tabular}

\begin{tabular}{lll}
\hline $\begin{array}{l}\text { Justus } \\
\text { Marquetand, } \\
\text { MD }\end{array}$ & $\begin{array}{l}\text { Department of Neurology } \\
\text { and Epileptology, Hertie } \\
\text { Institute of Clinical Brain } \\
\text { Research, University of } \\
\text { Tübingen, Germany }\end{array}$ & $\begin{array}{l}\text { Major role in the acquisition } \\
\text { of data, revised the } \\
\text { manuscript for intellectual } \\
\text { content }\end{array}$ \\
\hline $\begin{array}{l}\text { Christoph } \\
\text { Braun, PhD }\end{array}$ & $\begin{array}{l}\text { MEG-Center, University of } \\
\text { Tübingen, Germany; CIMeC, } \\
\text { Center for Mind/Brain } \\
\text { Sciences, University of } \\
\text { Trento, Italy; and }\end{array}$ & $\begin{array}{l}\text { Provided critical input on } \\
\text { the methods, project } \\
\text { supervision, revised the } \\
\text { manuscript for intellectual } \\
\text { content }\end{array}$ \\
& $\begin{array}{l}\text { and Epileptology, Hertie } \\
\text { Institute of Clinical Brain } \\
\text { Research, University of } \\
\text { Tübingen, Germany }\end{array}$ & \\
&
\end{tabular}


Appendix (continued)

\begin{tabular}{|c|c|c|}
\hline Name & Location & Contribution \\
\hline $\begin{array}{l}\text { Holger } \\
\text { Lerche, MD }\end{array}$ & $\begin{array}{l}\text { Department of Neurology } \\
\text { and Epileptology, Hertie } \\
\text { Institute of Clinical Brain } \\
\text { Research, University of } \\
\text { Tübingen, Germany }\end{array}$ & $\begin{array}{l}\text { Revised the manuscript for } \\
\text { intellectual content }\end{array}$ \\
\hline $\begin{array}{l}\text { Niels K. } \\
\text { Focke, MD }\end{array}$ & $\begin{array}{l}\text { Clinic of Clinical } \\
\text { Neurophysiology, University } \\
\text { Medical Center Göttingen; } \\
\text { and Department of } \\
\text { Neurology and Epileptology, } \\
\text { Hertie Institute of Clinical } \\
\text { Brain Research, University } \\
\text { of Tübingen, Germany }\end{array}$ & $\begin{array}{l}\text { Designed and } \\
\text { conceptualized the study, } \\
\text { project supervision, } \\
\text { interpreted the results, } \\
\text { revised the manuscript for } \\
\text { intellectual content }\end{array}$ \\
\hline
\end{tabular}

\section{References}

1. Jallon P, Latour P. Epidemiology of idiopathic generalized epilepsies. Epilepsia. 2005; 46(suppl 9):10-14

2. Scheffer IE, Berkovic S, Capovilla G, et al. ILAE classification of the epilepsies: position paper of the ILAE Commission for Classification and Terminology. Epilepsia. 2017;58(4):512-521.

3. Koeleman BP. What do genetic studies tell us about the heritable basis of common epilepsy? Polygenic or complex epilepsy? Neurosci Lett. 2018;667:10-16.

4. Berkovic SF, Howell RA, Hay DA, Hopper JL. Epilepsies in twins: genetics of the major epilepsy syndromes. Ann Neurol. 1998;43(4):435-445.

5. International League Against Epilepsy Consortium on Complex Epilepsies. Genomewide mega-analysis identifies 16 loci and highlights diverse biological mechanisms in the common epilepsies. Nat Commun. 2018;9(1):5269.

6. Gottesman II, Gould TD. The endophenotype concept in psychiatry: etymology and strategic intentions. Am J Psychiatry. 2003;160(4):636-645

7. Chowdhury FA, Elwes RD, Koutroumanidis M, Morris RG, Nashef L, Richardson MP. Impaired cognitive function in idiopathic generalized epilepsy and unaffected family members: an epilepsy endophenotype. Epilepsia. 2014;55(6):835-840.

8. Wandschneider B, Kopp U, Kliegel M, et al. Prospective memory in patients with juvenile myoclonic epilepsy and their healthy siblings. Neurology. 2010;75(24): 2161-2167.

9. Iqbal N, Caswell H, Muir R, et al. Neuropsychological profiles of patients with juvenile myoclonic epilepsy and their siblings: an extended study. Epilepsia. 2015;56(8): 1301-1308.

10. Wandschneider B, Hong S-J, Bernhardt BC, et al. Developmental MRI markers cosegregate juvenile patients with myoclonic epilepsy and their healthy siblings. Neurology. 2019;93(13):e1272-e1280.

11. Caciagli L, Wandschneider B, Xiao F, et al. Abnormal hippocampal structure and function in juvenile myoclonic epilepsy and unaffected siblings. Brain. 2019;142(9): 2670-2687.

12. Chowdhury FA, Woldman W, FitzGerald TH, et al. Revealing a brain network endophenotype in families with idiopathic generalised epilepsy. PLoS One. 2014; 9(10):e110136.

13. Caciagli L, Wandschneider B, Centeno M, et al. Motor hyperactivation during cog nitive tasks: an endophenotype of juvenile myoclonic epilepsy. Epilepsia. 2020;61(7): $1438-1452$.

14. Wandschneider B, Centeno M, Vollmar C, et al. Motor co-activation in siblings of patients with juvenile myoclonic epilepsy: an imaging endophenotype? Brain. 2014; 137(pt 9):2469-2479.

15. Li Hegner Y, Marquetand J, Elshahabi A, et al. Increased functional MEG connectivity as a hallmark of MRI-negative focal and generalized epilepsy. Brain Topogr. 2018; 31(5):863-874

16. Elshahabi A, Klamer S, Sahib AK, Lerche H, Braun C, Focke NK. Magnetoencephalography reveals a widespread increase in network connectivity in idiopathic/ genetic generalized epilepsy. PLoS One. 2015;10(9):e0138119.

17. Van Diessen E, Numan T, Van Dellen E, et al. Opportunities and methodological challenges in EEG and MEG resting state functional brain network research. Clin Neurophysiol. 2015;126(8):1468-1481.

18. Smit D, Posthuma D, Boomsma D, De Geus E. Heritability of background EEG across the power spectrum. Psychophysiology. 2005;42(6):691-697.

19. Colclough GL, Smith SM, Nichols TE, et al. The heritability of multi-modal connectivity in human brain activity. Elife. 2017;6(1):e20178.

20. Marquetand J, Vannoni S, Carboni M, et al. Reliability of MEG and hd-EEG restingstate functional connectivity metrics. Brain Connect. 2019;9(7):539-553.
21. Nolte G, Bai O, Wheaton L, Mari Z, Vorbach S, Hallett M. Identifying true brain interaction from EEG data using the imaginary part of coherency. Clin Neurophysiol. 2004;115(10):2292-2307.

22. Winkler AM, Webster MA, Vidaurre D, Nichols TE, Smith SM. Multi-level block permutation. Neuroimage. 2015;123:253-268.

23. Smith SM, Nichols TE. Threshold-free cluster enhancement: addressing problems of smoothing, threshold dependence and localisation in cluster inference. Neuroimage. 2009;44(1):83-98

24. Cohen J. A power primer. Psychol Bull. 1992;112(1):155.

25. Chen G, Taylor PA, Haller SP, et al. Intraclass correlation: improved modeling approaches and applications for neuroimaging. Hum Brain Mapp. 2018;39(3): 1187-1206.

26. Desikan RS, Ségonne F, Fischl B, et al. An automated labeling system for subdividing the human cerebral cortex on MRI scans into gyral based regions of interest. NeuroImage. 2006;31(3):968-980.

27. Niso G, Carrasco S, Gudín M, Maestú F, del-Pozo F, Pereda E. What graph theory actually tells us about resting state interictal MEG epileptic activity. NeuroImage. 2015; 8:503-515.

28. Clemens B, Puskás S, Besenyei M, et al. EEG-LORETA endophenotypes of the common idiopathic generalized epilepsy syndromes. Epilepsy Res. 2012;99:281-292

29. Jiruska P, De Curtis M, Jefferys JG, Schevon CA, Schiff SJ, Schindler K. Synchronization and desynchronization in epilepsy: controversies and hypotheses. J Physiol. 2013;591(4):787-797.

30. Tangwiriyasakul C, Perani S, Abela E, Carmichael DW, Richardson MP. Sensorimotor network hypersynchrony as an endophenotype in families with genetic generalized epilepsy: a resting-state functional magnetic resonance imaging study. Epilepsia. 2019; 60(10):e14-e19.

31. Tangwiriyasakul C, Perani S, Centeno M, et al. Dynamic brain network states in human generalized spike-wave discharges. Brain. 2018;141(1):2981-2994.

32. Gotman J, Grova C, Bagshaw A, Kobayashi E, Aghakhani Y, Dubeau F. Generalized epileptic discharges show thalamocortical activation and suspension of the default state of the brain. Proc Natl Acad Sci. 2005;102(42):15236-15240.

33. Larivière $\mathrm{S}$, Rodríguez-Cruces $\mathrm{R}$, Royer J, et al. Network-based atrophy modeling in the common epilepsies: a worldwide ENIGMA study. Sci Adv. 2020;6(47):eabc6457.

34. Whelan CD, Altmann A, Botía JA, et al. Structural brain abnormalities in the common epilepsies assessed in a worldwide ENIGMA study. Brain. 2018;141(2):391-408.

35. Pizzo F, Roehri N, Villalon SM, et al. Deep brain activities can be detected with magnetoencephalography. Nat Commun. 2019;10(1):1-13.

36. Focke NK, Diederich C, Helms G, Nitsche MA, Lerche H, Paulus W. Idiopathicgeneralized epilepsy shows profound white matter diffusion tensor imaging alterations. Hum Brain Mapp. 2014;35(7):3332-3342.

37. Van Beijsterveldt C, Molenaar P, De Geus E, Boomsma D. Heritability of human brain functioning as assessed by electroencephalography. Am J Hum Genet. 1996;58(3):562

38. Engel AK, Fries P. Beta-band oscillations: signalling the status quo? Curr Opin Neurobiol. 2010;20(2):156-165.

39. Kopell N, Ermentrout G, Whittington M, Traub R. Gamma rhythms and beta rhythms have different synchronization properties. Proc Natl Acad Sci. 2000;97(4):1867-1872.

40. Baumgarten TJ, Oeltzschner G, Hoogenboom N, Wittsack H-J, Schnitzler A, Lange J. Beta peak frequencies at rest correlate with endogenous $\mathrm{GABA}+/ \mathrm{Cr}$ concentrations in sensorimotor cortex areas. PLoS One. 2016;11(6):e0156829.

41. May P, Girard S, Harrer M, et al. Rare coding variants in genes encoding GABAA receptors in genetic generalised epilepsies: an exome-based case-control study. Lancet Neurol. 2018;17(8):699-708.

42. Edenberg HJ, Dick DM, Xuei X, et al. Variations in GABRA2, encoding the $\alpha 2$ subunit of the GABAA receptor, are associated with alcohol dependence and with brain oscillations. Am J Hum Genet. 2004;74(4):705-714.

43. Smit DJ, Wright MJ, Meyers JL, et al. Genome-wide association analysis links multiple psychiatric liability genes to oscillatory brain activity. Hum Brain Mapp. 2018;39(11) 4183-4195.

44. Meyers JL, Zhang J, Chorlian DB, et al. A genome-wide association study of interhemispheric theta EEG coherence: implications for neural connectivity and alcohol use behavior. Mol Psychiatry. 2020:1-13.

45. Tewarie P, Hunt BA, O'Neill GC, et al. Relationships between neuronal oscillatory amplitude and dynamic functional connectivity. Cereb Cortex. 2019;29(6):2668-2681.

46. Steinhoff BJ, Scholly J, Dentel C, Staack AM. Is routine electroencephalography (EEG) a useful biomarker for pharmacoresistant epilepsy? Epilepsia. 2013;54(suppl 2):63-66.

47. Clemens B, Piros P, Bessenyei M, Hollódy K. Lamotrigine decreases EEG synchronization in a use-dependent manner in patients with idiopathic generalized epilepsy. Clin Neurophysiol. 2007;118(1):910-917.

48. Vidaurre D, Hunt LT, Quinn AJ, et al. Spontaneous cortical activity transiently organises into frequency specific phase-coupling networks. Nat Commun. 2018;9(1):1-13.

49. Marini C, Scheffer IE, Crossland KM, et al. Genetic architecture of idiopathic generalized epilepsy: clinical genetic analysis of 55 multiplex families. Epilepsia. 2004; 45(5):467-478

50. Tashkandi M, Baarma D, Tricco AC, Boelman C, Alkhater R, Minassian BA. EEG of asymptomatic first-degree relatives of patients with juvenile myoclonic, childhood absence and rolandic epilepsy: a systematic review and meta-analysis. Epileptic Disord. 2019;21(1):30-41. 


\section{Neurology}

\section{Heritability of Magnetoencephalography Phenotypes Among Patients With Genetic Generalized Epilepsy and Their Siblings}

Christina Stier, Adham Elshahabi, Yiwen Li Hegner, et al.

Neurology 2021;97;e166-e177 Published Online before print May 27, 2021

DOI 10.1212/WNL.0000000000012144

This information is current as of May 27, 2021

\section{Updated Information \&} Services

\section{References}

Subspecialty Collections

Permissions \& Licensing

Reprints including high resolution figures, can be found at: http://n.neurology.org/content/97/2/e166.full

This article cites 49 articles, 5 of which you can access for free at: http://n.neurology.org/content/97/2/e166.full\#ref-list-1

This article, along with others on similar topics, appears in the following collection(s):

Functional neuroimaging

http://n.neurology.org/cgi/collection/functional_neuroimaging

Information about reproducing this article in parts (figures,tables) or in its entirety can be found online at:

http://www.neurology.org/about/about_the_journal\#permissions

Information about ordering reprints can be found online:

http://n.neurology.org/subscribers/advertise

Neurology ${ }^{\circledR}$ is the official journal of the American Academy of Neurology. Published continuously since 1951, it is now a weekly with 48 issues per year. Copyright Copyright (C) 2021 The Author(s). Published by Wolters Kluwer Health, Inc. on behalf of the American Academy of Neurology.. All rights reserved. Print ISSN: 0028-3878. Online ISSN: 1526-632X.

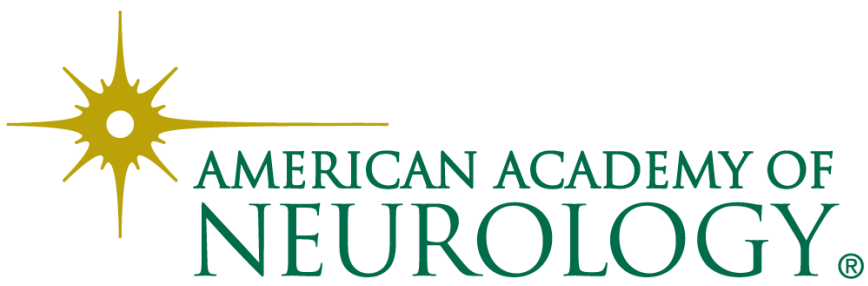

